\title{
User behaviour: searching for scholarly material using electronic resource discovery systems
}

\author{
Hanna Stelmaszewska $\quad$ B.L. William Wong \\ Interaction Design Centre, Middlesex University Learning \\ The Burroughs, Hendon London NW4 4BT \\ Nazlin Bhimani \\ Balbir Barn \\ Resources, Middlesex University \\ The Burroughs, Hendon London NW4 4BT \\ h.stelmaszewska / w.wong / n.bhimani / b.barn @ mdx.ac.uk
}

\begin{abstract}
This paper reports on user behaviour when interacting with various electronic resource discovery systems (ERDS) while searching for scholarly material. It focuses on the search strategies applied by 34 students and researchers in Business and Economics from three UK universities to find relevant information on a specified topic. The findings of the study are presented in relation to existing information-seeking models. Although there are a lot of similarities between existing models we have found some differences between those and our study. These are: the use of personal/ social networks to develop keywords for the queries as well as obtaining relevant material, study participants very rarely applied only one search strategy but tended to carry out combined searches which vary depending on the user group, and participants used different means of storing material. We expect these findings to help shape a set of requirements for next generation information discovery provisions in academic libraries.
\end{abstract}

User behaviour, information seeking, ERDS, searching, storage

\section{INTRODUCTION}

Information seeking activity is an important part of the development of academics and scholars who have access to many specialized electronic resource discovery systems. Electronic resource discovery systems (ERDS) refer to electronic databases, e-journals portals (such as EBSCO EJS, Emerald, ProQuest), federated search engines, catalogues, e-books and various electronic newspapers subscribed by higher education institutions.

Despite availability of these resources, scholars often bypass the library-provided resource discovery systems and use Google, Google Scholar or Wikipedia, which provide (as they claim) 'easy and quick' access and use. One way to address the usability issues of electronic resource discovery systems is to understand: how people develop their information seeking strategies; what they do with found information and how the current resources discovery systems support or hamper them.

Based on a qualitative study of 34 students and researchers in Business and Economics from across three universities in the UK, we report on our findings from investigations into these issues, and how this may help us in the design of a 'new generation' resource discovery systems. Such systems would need to support the information search and retrieval process across a large spectrum of users from novices, who require more support, to experts, who need a set of more sophisticated tools. In addition, those systems need to provide means of storing material with an easy and transparent access that can be available across variety of resources and platforms. The findings discussed in this paper, are based on a part of our study.

\subsection{Background of Information seeking}

Over the years a large body of literature has discussed how people search for information in electronic and paper resources $([2,3,4,6,7,14,16,17,20,21$, $28,29,33])$. Researchers have focused on a number of issues: how people manage their bookmarks [1]; what tasks people perform using the Web [25] and how people keep track of information on the Web [12]. Notably, Jones et al. [9] looked at how Web information is stored by observing user interaction when using email, files and the Web. However, they focused specifically on how people used files and email to support their Web interactions. Another group of researchers concentrated on investigating the effectiveness of exploratory search systems([[8, $15,24])$ whereas information behaviour in context was thesubject of research for $([10,11,30)$,$] .$

Vakkari's [29] longitudinal study focused on the development within one extended searching episode. He argues that the subject knowledge plays an important role in users' query reformulation and it guides their choice of options provided by the system.

Based on her observations of students working on an assignment in a physical library, Kuhlthau [14] describes the information search process as a series of stages that include initiation, selection, exploration, formulation, collection and 
presentation. Ellis' [5] early model of information seeking behaviours is based on observations of the information seeking activities of social scientists. $\mathrm{He}$ identified six characteristics of information seeking patterns: starting, chaining, browsing, differentiating, monitoring, and extracting. Ellis noted that, 'the detailed interrelation or interaction of the features in any individual information seeking pattern will depend on the unique circumstances of the information seeking activities of the person concerned at that particular point in time' ([5, p.178]). This work was then extended by two more stages: verifying and ending, as a result of a study on the information seeking activities of research physicists and chemists $([5,7])$ A similar model, although perhaps better for an electronic environment was proposed by Marchionini [16]. The model identifies a sequence of eight action-oriented tasks developed in parallel: recognition of a problem, definition of the problem, selection of information resources, query formulation, search execution, results examination, information extraction and information search reflection, iteration and completion. Unlike Ellis' [5], this model presents different processes occurring during information seeing rather then the behaviour of individual information seekers. Bates [2] has described the information seeking process more from a strategic point of view whereas Wilson [33] viewed it as task processes. The latter model's places emphasis on stages in the problem solving process, which occurs in a linear manner. These stages constitute: problem identification, problem definition, problem resolution, and solution statement. A similar model has been proposed by Sutcliffe and Ennis [28]. This work supports the development of a cognitive model of information retrieval and also consists of four activities: problem identification, need articulation, query formulation, and results evaluation.

These models are usually not intended to fully model all possible enumerations of user behaviour. Instead the models describe the more frequent and predictable aspects of user behaviour.

For instance, O'Day \& Jeffries, [17] use the term information orienteering that describe search strategies where users apply information from their current search to help identify the next step of information searching rather then trying to find information by writing one long complex query specifying all information needs. Pirolli and Card $([20,21])$ suggest different approaches to understanding information seeking: 'information foraging theory' and 'information scent'. The former adopted the concept from a food foraging theory called Optimal Foraging Theory in order to explain human information-seeking and sense-making behaviour. The information foraging theory attempts to anticipate people's strategies for navigating with information structures. Furthermore, the theory assumes that in modern world people use their various thinking strategies such as identification, categorization to explore, find and eventually 'consume' information [22]. Information foraging refers to activities associated with assessing, seeking, and handling information sources. Such search will be adaptive to the extent that it makes optimal use of knowledge about expected information value and expected costs of accessing and extracting the relevant information. Information scent refers to the recognition and the use of cues such as links within World Wide Web or bibliographic citations that provide users with information about the content, which is not visible at the current stage. They argue that users perceive relevance of information based on some measures of information scent, which are usually derived based on statistical techniques that extract semantic relatedness of words from large text databases An extension to the Pirolli \& Card [20] information foraging theory is the model of a sense making loop for intelligence analysis [21]. The authors suggest that the overall process is organized into two major loops of activities: (i) a foraging loop that involves processes aimed at seeking information, searching and filtering it, as well as reading and extracting information possibly into same schema; (ii) a sense making loop that involves iterative development of a mental model (conceptualization) from the schema that best fits the evidence.

The sense-making model offers a novel and useful perspective on designing systems for information retrieval. It encourages the designer to think about the structure of the interface, how to support different searching and browsing strategies appropriate for the context of work and how to effectively use metadata cues to enhance item selection and patch navigation.

Recent work by Spencer [26] presents a practical view on people searching for information. Based on her observations of users in their natural settings, Spencer argues that people experience four different modes during their seeking information activity: (i) "known-item" search where people know what they want, what search terms to use and what resources will provide them with information; (ii) "exploratory" search where people have some idea of what they need but their search terms may not be the right once and they may not know which resources to use. However, when they do find information, they will recognise it if it is the right information; (iii) "don't know what you need to know" search where people may or may not know how to articulate what they need but will recognize when they have found the right (possibly partial) answer; and (iv) "re-finding" where people look for things they have seen before, i.e. hunting down information they have previously seen. 
These models have provided us with a starting point about what people do when looking for information in electronic environments. While there are many similarities between the processes we found and the processes described in the models above, we have found some differences. These are: (i) the use of personal/social networks in order to develop a set of keywords for the queries, (ii) the use of combined searches instead of one search (e.g. 'Simple Search') that vary across different user groups, and (iii) the ways users store information. Another element that is implied but not explicit in the above models is the 'Prior Knowledge and Experience' (PK/E) that a user draws on with when beginning a search. We believe this study provides a better understanding of what scholars do when seeking academic material using various electronic resource discovery systems. In addition, it also determines how the current systems support or hinder user searching activities and strategies as well as storing of found material, all of which can be used to inform the development of new systems better suited to meet user requirements.

\section{METHOD}

The study adopted a qualitative approach to obtain a better understanding of how scholars search for high quality academic materials.

We recruited 34 volunteers studying or researching topics in business and economics from three categories of British universities: the large research intensive universities, the smaller research-led universities, and the former polytechnics. Volunteers represented undergraduate (UG), postgraduate (PG) (typically PhD students) and expert researchers (EX) (typically post-docs). There were altogether 16 female and 18 male participants, aged between 22-55 years (Table 1). Participants were given pseudo-names (e.g. MP10, LP2, CP4) for reasons of anonymity. The intention in working with these groups was to have a mix of users who would be expected to have different levels of understanding of the various resource discovery systems, which they would use when searching for quality scholarly materials.

\begin{tabular}{llcccc}
\hline & & $\begin{array}{c}\text { Under- } \\
\text { grads }\end{array}$ & $\begin{array}{c}\text { Post- } \\
\text { grads }\end{array}$ & $\begin{array}{c}\text { Resear } \\
\text { chers }\end{array}$ & Total \\
\hline Stage 1: Focus Groups & 6 & 3 & 0 & 9 \\
\hline $\begin{array}{l}\text { Stage 2: } \\
\text { Observations } \\
\text { and in-depth } \\
\text { interviews }\end{array}$ & $\begin{array}{l}\text { Large research } \\
\text { intensive university }\end{array}$ & 5 & 4 & 3 & 12 \\
\cline { 2 - 6 } & Former polytechnic & 5 & 3 & 4 & 12 \\
\cline { 2 - 6 } & $\begin{array}{l}\text { Smaller research- } \\
\text { led university }\end{array}$ & 0 & 6 & 4 & 10 \\
\cline { 2 - 6 } & Total & 10 & 13 & 11 & 34 \\
\hline
\end{tabular}

Table 1. Distribution of participants across universities

The study was initiated with two focus groups (9 students) in order to learn about the language and concepts used by them when searching online for scholarly material. Data gathered helped us to develop three task scenarios of varying levels of difficulty and ambiguity that were used in the next stage of the study. Focus groups are a qualitative method, which can be used alone or with other qualitative or quantitative methods to improve the depth of understanding the needs and requirements of users and customers ([19, 31]).

The next stage of the study involved all 34 participants and consisted of 2-hour observation and 'think aloud' studies followed by in-depth interviews using Cognitive Task Analysis techniques such as Critical Decision Method and Cued Recall. User observation and think aloud methods where used to investigate what people do, how they do it and why when searching for information using various resource discovery systems. These tasks were used to observe searching behaviour and increased in levels of ambiguity and difficulty with task 1 being the simplest and task 3 the most difficult. The tasks are briefly presented below:

Task 1: Find a range of examples from film and television programs, which illustrate product placement 'in action'. Task 2: Find evidence of film tourism from a range of different film industries to illustrate the impact this may have had on tourism. Task 3: Imagine that you are the brand manager for a new range of mobile phones for Nokia; you required to produce evidence to demonstrate how you might use the film/television medium as a way of reaching your target audience.

Each session lasted between 40 - 80 minutes was screen and audio recorded and later transcribed for analysis.

Following each observation session we carried out an in-depth interview that provided supportive evidence for the observation. We adapted the Critical Decision Method (CDM) ([9, 13, 35]), an open-ended, semi-structured retrospective interview method, to investigate the nature of information seeking, strategies used, decisions made and problems arising when participants were searching for information. CDM was combined with another cognitive task analysis technique called Cued Recall $([18,23])$. The Cued Recall technique helped us further probe aspects of the participants' interaction that we did not understand or had doubts about. The interviews focused on the following: (i) identifying the expertise and underlying rationale for the search behaviour demonstrated during the observation session; (ii) problems and difficulties users experienced (iii) clarifying ambiguity that occurred during the observation session; and (iv) exploring the differences between services and attributes of physical and electronic libraries. Each interview lasted between $40-60$ minutes was audio recorded and transcribed for subsequent analysis. For the analysis part of the study, we used 
approaches such as Emergent Themes Analysis (ETA), a technique for rapid yet systematic and rigorous extraction of key themes from both the observation and interview data sets of individual participants.

The ETA approach is a technique for qualitative data analysis that is based on Grounded Theory but takes advantage of the exploratory and efficient CDM data collection method [35]. ETA uses a concept distillation process to rapidly and systematically identify broad themes that are similar in ideas and concepts reported across interviews or observations. The data can then be identified, indexed and collated. The themes emerge from the data and so strengthen the validity of the findings. Using the same procedure, the sub-themes or specific themes within the data are identified and further categorized. These categories form the basis for analysis. The themes were collated and analyzed across the different study groups and across all three institutions.

\section{FINDINGS AND DISCUSSION}

We found that when scholars search for academic material they go through a number of stages applying different behaviours and strategies at each stage to complete a particular task.

In brief, the process starts with Initiate, stage when users define the subject, understand the concepts and decide on the resources and search terms to be used, Next, participants proceed to the Search stage where they activate various search functions. These will depend upon on their mode of information search (such as Spencer's [26] 'know-item', 'exploratory', "don't know what you need to know" and "re-finding"). Tools such as 'simple search', Boolean search, links, and others offered by the systems are used in this situation. When a search returns a List, participants view and Evaluate them. The list is often scanned looking for the appearance of the search terms within a title, text displayed, checking the source of material for its credibility as well as the type of publication and its currency. In so doing, the user also evaluates the results for relevance. Based on the outcome of the 'Evaluation' a number of different actions can be taken. These can include: (i) 'Re-fine or re-formulate' search terms in the case of a search not been successful; (ii) 'Select and Review' when the user identifies a potentially relevant article. At this point, the relevance and usefulness of a specific item is assessed by the user, and followed by one of the decisions: discard, not sure, maybe view later, and keep; (iii) 'Store' if material is relevant, (iv) Abandon the search due to the lack of relevant results, not being able to access either the resource or an individual material, or a paper that required purchasing in order to view the full-text and participants were not prepared to do so, (v) 'Change the resource' when users decide or believe that the resource they have been using is no longer appropriate. At this point very often a 'phase shift' occurs, where the user switches resources and search behaviours. Detailed descriptions of each stage presented above have also been discussed elsewhere [29].

Despite the similarities between the behaviour displayed by scholars in our study and those described by the information seeking models discussed earlier, our findings present significant differences that are discussed next.

\subsection{Initiate behaviour and strategies}

This stage begins the information seeking process and although it has been discussed by others ([5, 14]) our addition to the activities embedded at this stage is that users utilize their personal/social networks (e.g. friends, associates, colleagues) as another way of identifying relevant concepts from which appropriate search terms or phrases can be generated.

CP2 (EX) commented, "I would go outside the academic [world] ...". Using peers to help them to remember or to suggest films with product placement examples came up many times - both by UG/PG students and researches. MP4 (UG) "...I have found stuff from ... [other] students"; LP2 (UG) "I will ask Mark Kecko [author of the article] for some information" and "I want to find film locations so I will go to the chat room or something to see what people are saying as it's easier to find ..." CP5 (PG).

Investigations by Ellis found that users relied on computerized searches to approach a new topic within an unknown field', but reported that both the scientists and engineers relied on expert intermediaries for this early stage of what he calls 'surveying' [5]. Watson-Bonne [32] and Ingwersen and Järvelin [11] also suggest the importance of the professional network in finding information. However, the emphasis is on the use of professional network not necessarily on the personal/social network as reported in this study, which the study groups used to identify relevant concepts and develop keywords. Another difference is the use of personal knowledge/ experience when making decisions about resources to be used. Participants often choose resources they are familiar with rather then looking for the best one. LP7 (EX) stated: "Let me try the beta catalogue. I remember that this was useful for wide searches". CP7, a researcher goes to Wikipedia as the first port of call: "I am going to Wikipedia ... [as it will refer me to] other sources". LP7 uses this for the same reasons, "I look at Wikipedia because often there are examples there and links that might be useful". This is an example of where Wikipedia is used to find references to other, maybe more reliable sources of information. CP8 (PG) says, “. . . first l'll go to Google 
and try to find because Google is the first thing that comes to my mind". This was both out of habit and often to gauge how the search hits on Google can help with an understanding of the subject. It is also a means to harvest keywords. For example, one of the students expressed concerns about the keywords and suggested his way of solving this problem: "If the keywords are not clear, I go to Google or electronic dictionaries to clarify [them]". (CP9, PG). "Even though I forbid my students to use Wikipedia, it is always the first place that I go to look for stuff. Mainly it is just to have a quick idea of what it is all about, and secondly, very often you have a list of first references where you can look for more information" (MP6, EX).

\subsection{Search behaviour and strategies}

Search activities have been a topic for discussions for many researchers $([5,16,20,21,28])$. However, the description of the ways and strategies people apply when searching for information are limited. This study offers a detailed account of the different search strategies employed. We found that the use of searching strategies depends on the participants' information literacy skills, their domain knowledge and their understanding of the task they were given. Participants utilized twelve different searches:

a) Simple Search (SS) - terms were taken from the given task and were used as a part of the search sentence and entered into the single search field (e.g. 'product placement in films'; or 'product placement in films and movies for the purpose of brand awareness'); b) Advanced Search (AD) - a number of search terms used together with different Boolean operators (e.g. AND, OR, AND NOT (e.g. 'television OR movie OR film AND product placement AN Times AND NOT Irish'); c) Simple and Advanced (SS\&AD) - a combination of the two described above. d) Personal Knowledge and Experience Search (PK/E) - terms are developed based on personal knowledge/experience such as the name of movie that participants have watched: 'yaaden full movie and coca cola', 'transformers 2 and Egypt' ' matrix and nokia' etc; e) Re-formulated Search (RFS) - terms were taken / combined from document keywords found in earlier searches and used to refine/re-formulate the search (e.g. 'galician mary-lou "product placement" Ise'); f) Link Search (LS) - follow the hyper-links within documents to find relevant information (e.g. 'product placement in television' => 'list of results' => 'wikipedia article' => 'TV programmes'); g) Multi-Field Search (MFS) - a number of search terms specified for different fields (e.g. 'film' in 'Citations and document text'; 'product placement' in 'title' AND 'tourism' in 'title') and carried out within the same search; h) Phrase Search (PHS) - terms were put in quotation marks to find information containing an exact phrase (e.g. "product placement"); i) Database Search (DBS) - specific databases were selected in order to narrow a search (e.g. EBSCO's Film and Television Literature Index); j) System Suggestions - follow system's suggestion to narrow the search by using 'Suggested Topics', 'Results by Source', 'Source Type', Clustered Results, Cited by and specific resources (e.g. Scholarly journals, peer review) and/ or correct spelling or take on suggested keyword, e.g. "Do you mean ... ?"; k) Full Text/PDF Search (FT/Pdf) - select Full Text or Pdf file options to narrow the results; I) Date Limit Search (DL) - refine results by date range e.g. 2006 ${ }^{\circ}$ current year.

Although the above presents a list of search types, participants rarely used only one search (e.g. Simple Search). Instead, the evidence suggests that a combination of search types were used in any one searching session. For instance: participants who applied 'Link Search' (i.e. clicking on a link to investigate further the potential value of a reference) during their information seeking activities also pursued 'Re-formulated Search' (using keywords obtained from the retrieved documents to do a new search) as well as 'Multi-Field Search' where a 'publication' or 'citation in a document text' were used in selective fields (e.g. 'mobile phone' in 'Document title', 'advertising' in 'Citation and document text', 'marketing' in 'Citation and document text'). The same participants also invoked their personal knowledge and experience (PK/E) in the subject area to provide new leads in the search. This was mostly observed in the context of task 1 and 2, where participants were asked to provide examples of 'product placement' and evidence of 'film tourism'. Their queries included the name of products, the title of movies or names of places of which they were aware. The use of individual searches by different user groups is discussed elsewhere [36].

These searches were applied in an iterative rather then linear manner, which is in line with Sutcliffe and Ennis [28]. They identify four knowledge sources - the domain, the resources, thedevice, and information retrieval skill - which, together with ataxonomy of information need, may be used to predict the user'sbehaviour. The view of the effect of the domain knowledge on thequery formulation was also shared by Vakkari [29] and the study reported here confirmed these observations.

When discussing the motivation and use of different searches O'Day \& Jeffries [17] defined a number of triggers that motivate a seeker to switch from one search strategy to another. Thesetriggers include: (i) the completion of one step and beginning ofthe next logical step in a plan, (ii) encountering something interesting that provides a new way of thinking about a topic ofinterest, or a new, interesting angle to explain a topic or problem,

(iii) encountering a change or violation of previous expectationsthat requires further investigation, 
and (iv) encountering inconsistencies with or gaps in previous understanding that requires further investigation. In addition, O'Day \& Jeffries [17] suggested stop conditions i.e. circumstances under which peopledecided to stop searching. These were: (i) there were no morecompelling triggers, (ii) an 'appropriate amount of material had been found, or (iii) discovering a market was too small to beworth researching. Our study observed similar behaviours during the search process (see Section 3).

\subsection{Search grouping strategies}

It was observed that most of the time users combined different searches. Five most frequently occurring search groupings have been identified, they are:

a) The Link Search Grouping: Re-formulated Search, Multi-Field Search, Personal Knowledge and Experience

b) The Re-formulated Search Grouping: System Suggestion, Multi-Field Search, Link Search

c) The Multi-Field Search Grouping: Re-formulated Search, Link Search, System Suggestions

d) The System Suggestion Grouping: Re-formulated Search, Multi-Field Search

e) The Database Selection Grouping: Advanced Search

It should be noted that all of these groupings have the Simple Search as common, i.e. the Simple Search is the fundamental building block on which the other searches build. These groupings of searches were used during the information seeking activities. It is important to understand that the sequence in which they appear is not as relevant as the fact that they are likely to be used as a suite of actions. Moreover, when users applied them they often changed between resources e.g. from Google they may go to the library or by following a hyperlink they may open a website, which will appear in a new tab. Consequently, when this happens they often lose the flow of the process or find themselves logged-out of the system, which means that they have to start the process again. Users expressed frustration and annoyance when this happened: "Another issue with ProQuest is 'Time Out'. It is irritating because all the searches are lost!" (CP4, EX).

What is required from the electronic resource discovery systems (ERDS) is the ability to automatically save all searches performed during a particular session. Although, increasingly ERDS have this option built in, it still requires the user to select this option after each search. Moreover, the current ERDS do not support a seamless transition between different resources.

The Link Search Grouping incorporates the 'Reformulated Search', 'Multi-Field Search' and 'Personal Knowledge/Experience' search. This search was used most frequently by the PG group and less frequently by Experts and by the UG group.
Participants followed links in order to find more information, to confirm previous findings or simply to explore other possibly related material (e.g. follow references). Following hyper-links were perceived to be time consuming and Experts preferred to be more focused on their task and performed searches that resulted in immediate outcomes. Within this grouping we observed that participants, for instance CP4 (EX) applied her personal knowledge of a place she had visited, which was connected to a movie she had watched (PK/E). She used this as a starting point to look for answers for task 2.

Extract from the observation and think aloud:

She starts with Google as she knows some places that have impact on tourism. She puts 'New Zealand' (241,000,000 results)

\begin{abstract}
CP4: 'I remember when I was there they have lots of stuff there. I know what l'm looking for ... it's the official tourism web site for NZ and l've used it before so it will be quick. Ether I have something or I won't but I'll be quick.'
\end{abstract}

CP4 opens the official NZ tourist web site She then searches within the site for 'Lord of the Ring' -102 results
CP4: ' l've already found relevant information so I'm very pleased with myself. There is so much in here. I'll be very chuffed with this.'

During the evaluation process CP4 found search terms (a name of an organization supporting film tourism in UK and also a name of the site promoting tourism in Scotland). This was in relation to a movie that was made in Scotland, which she used for the consecutive search (Re-Formulated Search).

Extract from the observation and think aloud:

\begin{abstract}
CP4:'There is something here about 'Film Council' so I'm going to have a look at the 'Film council' and see if I can see anything about it.'
\end{abstract}

She goes to Google and searches for 'film council'(RFS)

This user also followed links (Link Search) in resulting documents (an article about promoting film tourism in UK), which helped her in expanding the search terms as well as suggesting and combining multiple fields (MFS), in which to search for information. In this case, the search was within 'a title' (MFS) as she was looking for a specific journal that her previous search retrieved and she wanted to explore more within this journal.

Re-formulated Search Grouping. The most popular searches of this grouping were: 'System Suggestions', 'Multi-Field Search' and 'Link Search'. Participants who extracted search terms from retrieved documents and used them in their consecutive searches were also more inclined to combine their 'RFS' with 'System Suggestions' 
where they used system help such as 'Suggested Topics, 'Results by Source', Source Type' or selected specific resources (e.g. Scholarly journals, peer review) to narrow the search. Participants in this grouping also followed links in resulting documents (LS), which helped them expanding their search terms as well as suggesting and combining multiple fields in which to search (MFS).

Extract from the observation and think aloud of LP2 (UG):

LP2 uses information from the results about MaryLou Galician and performs a new search: Google 'Mary-Lou Galician Arizona State University' - 2,420 results. She works through the results and opens one document in a new tab. After reading the page LP2 decides to follow a link (Link Search) to 'Handbook of Product Placement in the Mass Media: News Strategies in Marketing, Theory, Practice, Trends and Ethics' which takes her to a new location - 'Taylor \& Francis' page.

From the study observations, it seems that Experts mostly used this combination of searches. It may be suggested that their greater familiarity with different ERDS environments as well as knowledge of a subject domain allowed them to be more 'adventurous' with their choice of searches.

Multi-Field Search Grouping consists of 'Reformulated Search', 'Link Search' and 'System Suggestions' components. Users who selected a number of search terms in different fields (MFS) are said to be users of the 'Multi-Field Search' action.

Extract from the observation and think aloud of CP1 (EX):

CP1 goes to Google Scholar and puts 'product placement' -719 results ... She finds an article that she wants to view but it is a 'citation' not a link to a document. She goes to the Library Resources and selects EBSCO and Business Source Complete. Next she puts the title of the paper into the search box and selects in 'Title' (MFS)

These MFS users applied this search in conjunction with 'Re•formulated Search', 'System Suggestions' and 'Link Search'. It appears that MFS by narrowing down the results provides 'System Suggestions' that are more relevant to the search, which consequently delivers more focused, topic driven results. In addition, users in this grouping also used their skills to extract new terms from the search documents (RFS), which opened new possibilities for them in terms of searches but also Link Search that expanded their original searches. Experts used this grouping more often than other groups, which may suggest that the higher level of familiarity with ERDS in following up 'System Suggestions' gives participants a sense of making progress and 'going in the right direction'.

System Suggestion Search Grouping includes Reformulated and Multi-Field Search. Participants who were more familiar and experienced in working with different ERDS followed the help provided by a system ('System Suggestions') in a form of 'Results by Source' or 'Suggested Topic', which allowed them to narrow their searches to the areas selected by a resource discovery system.

Extract from the observation and think aloud of CP8 (EX):

CP8 goes to ProQuest, selects 'All Databases' and puts 'product placement in action' - 159 results.

CP8: "Now when I see 'Suggested Topics' such as 'Product Placement and Television Programs' because I have to find examples from television programs I just click on that to look if there are good articles on that".

It seems that the new results obtained from the 'System Suggestions' provides new terms helping the users to re-formulate their queries (RFS). In addition, users in this grouping took advantage of different field options (MFS), which offered ways to narrow the search results further. This search grouping behaviour was popular amongst Experts who are more advanced in query formulation using other sources than those given in the tasks as well as following 'System Suggestions' and using advanced features of resources discovery systems (i.e. MFS). Database Selection Search Grouping incorporates Database Selection Search and Boolean Search. Users who selected specific databases in order to narrow a search (e.g. EBSCO's Film and Television Literature Index) also took advantage of a Boolean operator AND, OR, or AND NOT to limit not only the scope of their search but also to bring together the results for a number of searches within one action (e.g. 'television' OR 'movie' OR 'film' AND 'product placement' AND 'Times'). It seems that combining these two searches saved time but more pertinently provided fewer but more relevant results. The researchers (EX) from a small research-led university were more inclined to use these combinations of searches. The participants seemed to be more aware of the different databases available from the library resources as well as taking advantage of Boolean Search provided by the resources they used during the information seeking activities.

Extract for the observation and think aloud of CP4 (EX):

CP4:"Normally, I use Inform Global but because it's asking for examples ... I'm going to put newspapers". She then selects 'Databases' from the options and adds 'INFORM GLOBAL' and 'NEWSPAPERS'.

She then opens 'Advanced Search' and puts 'film' in 'Citations and document text' AND 'adverts' in 'Citations and document text'.

Another group of users who appeared to have knowledge about the databases subscribed to by the library and used Boolean Search options 
supported by EBSCO was the PG group from former polytechnic.

A key message for designers of ERDS is that this combination of searches is used in a substantive manner and designers need to facilitate and support this kind of search activity in the best possible way.

\subsection{Storage behaviour and strategies}

The study shows that storage of information is an important part of the information search and retrieval process. It takes place at the different stages of information seeking: (i) at the initial stage of evaluating results where participants scan through the list and select potentially relevant results for further evaluation. Information is either stored on separate tabs or files are downloaded and kept on the desktop, (ii) at the level of evaluation when participants work with individual document/material. All relevant material was stored. In the case of information being relevant, the material is stored using different means such as: a Word document for further references (i.e. details of documents/web sites were copy \& paste), systems' features such as 'My Research', 'Mark up', RefWorks or EndNote, bookmark, save in folders, or print.

The data shows that more traditional ways of storing material (i.e. printing) was used by students as well as researchers. They often preferred to print out their material for future referencing and reading. Printed material helped them to organize their thoughts and concepts as well as giving the flexibility of reading anytime anywhere. CP9 (PG) commented: "If it is more than 5 pages I will save and print it so I can read when travelling". However, some students preferred reading on-line. The reason for that was twofold: one related to the economic situation of international students; printing is expensive and they have to economize, another was participants' concern for the environment. CP6 (PG) commented: "I don't like to print because of concern for the environment" or CP10 (EX): "I do not print any more. It's bad for the environment and difficult to organize printed copies". MP12(EX) also commented: "I try not to print too much because it is a waste of paper". Others commented that reading on-line is something that they are used to doing: MP2(PG): "I'm used to reading on the screen ... it's better to read online" . Tabs were also used as a form of storing material. This was observed to be a common practice across all user groups. In addition, the specific reasons for using tabs are: (i) to run multiple searches using different resources, and therefore have the ease of speedily returning to an earlier search; (ii) to keep track of already viewed resources and material that could be used for future references; and (iii) to store information temporarily so they could go back and review it before storing it in an allocated place such as a folder, bookmark, print out or a Word document. This is consistent with Jones, Dumais \& Bruce [12]. Their study shows that people use different ways to gather or 'keep' their information. For instance, send emails to oneself, send emails to others, print, save documents as files, paste URL's into documents, put documents into a personal website, create bookmarks, write paper notes, create toolbar links, and use the note facility in Microsoft Outlook. It was apparent that there are new means of storing information, which are supported by different systems, however they are not always user friendly and required further improvements in order to support this process more efficiently. Pirolli and Card [21] also discussed the use of storage within their model of analysts' sense making. They suggest that storage of results occurs at two stages: one is the 'search and filter' stage where the relevant results are stored in 'shoebox' for further processing and this is similar to the 'tabbing' temporal storage observed in our study; the other storage happens at the 'read and extract' stage where the results from the 'shoebox' are read and the relevant snippets are stored in 'evidence files'. This corresponds to storing material in folders, as bookmarks or 'My Basket' in our study. It is evident that when seeking information storage plays an important role. However, the study groups used the storage features provided by resource discovery systems sporadically, as the majority of the users were not aware of their existence or how to use them. The current systems lack good ways of storing and retrieving documents allowing the users to create repositories of information that can be accessed easily and can be transferable across different resources. This might be another suggestion for user requirements for future electronic resource discovery systems.

\section{CONCLUSIONS}

In this paper we have reported findings from our qualitative study conducted with scholars (students and researchers from Business and Economics discipline) from three UK universities searching for quality material using various ERDS. The findings have focused on the differences between the behaviour displayed by our study group and those presented in previous work on information seeking. It was observed that people use personal/social networks at the Initiate stage of information seeking in order to understand the concept, find information and also develop the keywords.

The study also revealed that participants very rarely applied only one search (e.g. Simple Search) but that their strategy changed during the information seeking process in relation to the results obtained; users performed another search, refined or reformulated search, abandon a search or resource, 
or they changed resource. Most users tended to carry out combined searches (e.g. re-formulated searches where terms or concepts extracted from a document were used to pursue a new search). This search was often combined with a link search that gave the opportunity to follow hyper-links and extract new queries that were used as an input for the search terms in multiple fields. The important message here is that people used these different combinations of searches depending on their experience and familiarity with various ERDS and domain knowledge. However, the current systems do not support these various forms of searching making the transformation between them not as 'seamless' as it should be and the search process consequently becomes more difficult and time consuming. Providing users with a 'seamless' experience when moving from one system to another, whilst at the same time keeping a log of their search activities could be considered an enhancement in future design of resource discovery systems. Enabling users to see a log of their searching in real-time and to be given searching tips whilst searching will facilitate learning within resource discovery systems. This will allow novice users to learn the best ways of interrogating a system enabling them to see their errors and improve their information searching skills. Additionally, enabling users to set their individual search preferences will allow novice and expert users to interrogate systems in ways that they are comfortable with rather than having system-specific ways of searching imposed on them.

Another important issue that emerged from this study was about storing of information. The groups we studied used different means of storing the material they found depending on the stage they were at. At the stage of results' evaluation, they store material temporarily using the web browser's tabs system. These tabs are then re-visited for quick evaluation and then the information may be stored permanently using other means (e.g. notes in a Word document, save downloading material into a folder, bookmarking using the browser's feature). In addition, more sophisticated features provided by various resource discovery systems such as 'RefWorks', 'EndNote' and 'My Research' are rarely used for storing of information as the users are not always aware of their existence and how they work. It appears that the current systems lack transparent and easy ways of storing and retrieving documents allowing the users to create repositories of information that can be accessed easily and be transferable across different resources. This might be another suggestion for user requirements for future electronic resource discovery systems.

In summary, based on the study findings a number of design suggestions can be proposed. These are: (i) Improve facilities to support dynamic searches, and the retrieval and re-access process.
We recommend that electronic resource discovery systems (a) need to be flexible to enable users to shift seamlessly between different resources and to return to a previous search. We call this continuity across different search modalities. (b) facilitate the various combinations of search activities, rather than just static or rigid pathways, recognizing that search activities occur in various combinations, rather than in pre-defined sequences; and

(c) enable easy transition between 'internal' resources (library catalogues, EBSCO, Science Direct), and 'external' resources (the Internet, Google, Google Scholar, YouTube); (ii) Provide facilities for manipulation of search results to support visual triage and workflow. (iii) Provide easy and implicit query formulation and filtering; (iv) Provide information patches and

scents that will help users to find and review the relevancy of information in a most effective way. More detailed descriptions of these suggested recommendations is presented elsewhere [36].

\section{ACKNOWLEDGMENTS}

This work was funded by a grant from JISC, Ref. Num. CSSERSA2/SERV ENHANCE.

\section{REFERENCES}

[1] Abrahams, D., Baecker, R., \& Chignell, M. (1998). Information archiving with bookmarks: personal Web space construction and organization. Proceeding of SIGCHI'98, pp. 41-48.

[2] Bates, M.J. (1990). Where should the person stop and the information search interface start? Information Processing \& Management, 26, 575-591.

[3] Card, S. K., Pirolli, P., Van Der Wege, M., Morrison, J. B., Reeder,

R. W., Schraedley, P. K., \& Boshart, J. (2001). Information scent as a driver of Web behavior graphs. Proceedings of the ACM CHI '01 pp.498-505 [4] Chi, Ed H. Peter Pirolli, Kim Chen, James Pitkow (2001). Using Information Scent to Model User Information Needs and Actions on the Web. Proceedings of ACM CHI'01 pp. 490-497. ACM Press,

[5] Ellis, D. (1989). A behavioural approach to information retrieval system design. Journal of Documentation, 45, 171-212.

[6] Ellis, D., Cox, D., \& Hall, K. (1993). A comparison of the information-seeking patterns of researchers in the physical and social science. Journal of Documentation, 49, 356-369.

[7] Ellis, D., \& Haugan, M. (1997). Modelling the information-seeking patterns of engineers and research scientists in an industrial environment. Journal of Documentation, 53(4), 384-403. 
[8] Hoeber, O., Yang, X, D. (2008). Evaluating WordBars in exploratory Web search scenarios. Information Processing \& Management, 44(2), 485510.

[9] Hoffman, R. R., Crandall, B., \& Shadbolt, N. (1998). Use of the Critical Decision Method to elicit expert knowledge: A case study in the methodology of Cognitive Task Analysis. Human Factors, 40(2), 254-276.

[10] Hyldegård, J. (2009). Beyond the search process -Exploring group members' information behaviour in context. Information Processing \& Management, 45. [11] Ingwersen, P., \& Järvelin, K. (2005). The turn: Integration of information seeking and retrieval in context. Springer.

[12] Jones, W., Dumais, S. \& Bruce, H. 2002. Once found, what then?: A study of 'keeping' behaviours in the personal use of web information. Proceeding of ASIST 2002, Philadelphia, Pennsylvania.

[13] Klein, G. A., Calderwood, R., \& Macgregor, D. (1989). Critical decision method for eliciting knowledge. IEEE Transactions on Systems, Man and Cybernetics, 19(3), 462-472.

[14] Kuhlthau, C. (1988). Longitudinal case studies of the information search process of users in libraries. Library and Information Science Research, 10.3, 257-304.

[15] Kulles, B., Shneiderman, B. (2008). Users can change their web search tactics: Design guidelines for categorized overviews. Information Processing \& Management, 44(2), 463-484.

[16] Marchionini, G. (1995). Information seeking in electronic environments. Cambridge University Press.

[17] O'Day, V. L., \& Jeffries, R. (1993) Orienteering in an information landscape: how information seekers get from here to there. In

Proceedings of the INTERCHI Conference on Human Factors in Computing Systems (CHI'93), Amsterdam, April 1993. IOS Press.

[18] Omodei, M. M., McLennan, J. P., \& Wearing, A. J. (2005). How expertise is applied in real-world dynamic environments: Head mounted video and cued recall as a methodology for studying routines of decision making. In T. Betsch \& S. Haberstrohe (Eds.), The routines of decision making (pp. 271288). Mahwah, NJ: Lawrence Erlbaum.

[19] Payne, J. (1999). Researching Health Needs. A Community Based Approach. Sage Publications, London.

[20] Pirolli, P. \& Card, S. K. (1999). Information foraging. Psychological Review, 106, pp. 643-675.

[21] Pirolli, P. \& Card, S. K. (2005). The Sensemaking Process and Leverage Points for Analyst Technology as Identified Through Cognitive Task Analysis. Accessible at: https://analysis.mitre.org/.../ Final.../206_Camera_Ready_Paper.pdf

[22] Pirolli, P. (2007). Information foraging theory: adaptive interaction with information. Oxford; New
York: Oxford University Press.

[23] Puff, R. C. (1982). Handbook of Research Methods in Human Memory and Cognition. Academic Press series in cognition and perception.

[24] Qu, Y., Furnas. (2008). Model-driven formative evaluation of exploratory search: A study under a sensemaking framework. Information Processing \& Management, 44(2), 534-555.

[25] Sellen, A.J., Murphy, R., Shaw, K.L. (2002), "How knowledge workers use the web", Proceedings of the SIGCHI'02. Vol. 2002 pp.227-34.

[26] Spencer, D. (2006). Four Modes of Seeking Information and How to Design for Them. Available at: http://www.boxesandarrows.com/view/four_ modes_of_seeking_info rmation_and_how_to_design_for_them.

[27] Stelmaszewska, H., Blandford, A., \& Buckanan, G. (2005) Designing to change users' information seeking behaviour: a case study. In Chen, S. and Magoulas, G. (Eds) Adaptive and Adaptable Hypermedia Systems.

[28] Sutcliffe, A., \& Ennis M. (1998). Towards a cognitive theory of information retrieval. Interacting with Computers, 10, 321-351.

[29] Vakkari, P. (2001). A theory of the task-based information retrieval process: a summary and generalisation of a longitudinal study. Journal of Documentation., 57(1), 44-60.

[30] Vakkari, P. (2003). Task-based information searching. In B. Cronin (Ed.), Annual Review of Information Science and Technology (Vol. 37, pp. 413-464): Medford, NJ.

[31] Vaughn, S., Schumm, J.S., \& Sinagub, J. (1996). Focus group interviews in education and psychology. Sage Publication, Thousand Oaks, CA,

[32] Watson-Bonne, R. (1994) The Information Needs and Habits of Humanities Scholars, Reference Quarterly, 34(2), 203-16.

[33] Wilson, T. D. (1999). Models in Information Behaviour Research. Journal of Documentation, 55 (3), 249-270.

[34] Wong, B. L. W. (2006). The Critical Decision Method. In W. Karwowski (Ed.), International Encyclopedia of Human Factors and Ergonomics (pp. 3067-3073). Boca Raton, FL: CRC Press.

[35] Wong, B.L.W. \& Blandford, A. (2002). Analysing Ambulance Dispatcher Decision Making: Trialing Emergent Themes Analysis. In HF2002, Human Factors Conference "Design for the whole person - integrating physical, cognitive and social aspects". Melbourne, Australia: A joint conference of the Ergonomics Society of Australia (ESA) and the Computer Human Interaction Special Interest Group (CHISIG).

[36] Wong, B. L. W, Stelmaszewska, H., Bhimani, N., Barn, S., \& Barn,

B. (2009). User Behaviour in Resource Discovery: Final Report. Available at: www.ubird.mdx.ac.uk. 